\title{
Basophil Functions During Type 2 Inflammation: Initiators, Regulators and Effectors
}

\author{
Mark C. Siracusa and David Artis* \\ Department of Pathobiology, University of Pennsylvania, Philadelphia, Pennsylvania, USA
}

\begin{abstract}
Recent studies have identified several previously unrecognized functions of basophils in multiple models of Th2 cytokine-dependent immunity and inflammation. In addition to their established role as effector cells in inflamed tissues, findings now indicate that basophils express MHC class II and co-stimulatory molecules, can migrate into draining lymph nodes, present antigen to naive $\mathrm{CD}^{+}{ }^{+} \mathrm{T}$ cells and promote $\mathrm{Th} 2$ cell differentiation. In this context, basophils have been shown to be critically important for the induction and propagation of Th2 cytokine responses following exposure to helminth parasites and allergens. This article reviews recent conceptual advances in our understanding of basophil biology in the context of allergy and helminth infection.
\end{abstract}

Key Words: Basophil, Th2 cells, allergic inflammation.

\section{INTRODUCTION}

Basophils are the least abundant granulocyte population, making up less than $1 \%$ of all circulating leukocytes. Originally identified by the German scientist Paul Ehrlich in 1879, more than a century passed before basophils were shown to have the ability to bind IgE and produce histamine [1]. Despite these findings, basophils were considered to be a redundant population of cells that represented circulating or immature mast cells [2]. This prevailing dogma persisted until recent technical advances allowed researchers to study in vivo basophil biology in greater depth.

Significant conceptual advances in the functional biology of basophils were driven by technological developments and coincided with the generation of interleukin-4 (IL-4)/eGFP reporter mouse models. These tools facilitated the discovery that eosinophils, mast cells and basophils acquired constitutive IL-4/eGFP expression during their development [3-5]. These studies also allowed researchers to establish the expression of surface molecules on resting and activated basophils, which allowed them to be distinguished from tissueresident mast cells. Shortly after a specific surface phenotype was established, methods of depleting basophil populations by targeting the high-affinity IgE receptor or the membrane glycoprotein CD200R3 were developed [6-9]. The ability to identify and deplete basophils in vivo allowed for a series of studies that defined basophils as non-redundant contributors that play critical roles in the induction, regulation and propagation of Th2 cytokine-mediated immune responses.

The purpose of this article is to highlight recent conceptual advances in understanding the functions of basophils. We describe the mediators of basophil activation and effector functions in response to both endogenous and exogenous signals. Further, we focus on recent reports demonstrating

*Address correspondence to this author at the Department of Microbiology and Department of Pathobiology, University of Pennsylvania, 314 Hill Pavilion, 380 S University Avenue, Philadelphia, Pennsylvania 19104, USA; Tel: 215-898-7920; Fax: 215-746-2295;

E-mail: dartis@ vet.upenn.edu that basophils function as antigen presenting cells (APCs) that provide a critical link between innate and adaptive forms of Th2 cytokine-mediated immunity and inflammation.

\section{BASOPHIL EFFECTOR FUNCTIONS}

Although recent studies have fundamentally altered the way we think about basophil biology, historically basophils are best known as effector cells that release pre-formed mediators in response to activation via surface bound $\operatorname{IgE}[1]$. Circulating basophils bind $\operatorname{IgE}$ through the high affinity $\operatorname{IgE}$ receptor FceRI and degranulate upon FceRI crosslinking [10, 11]. Basophils activated via surface bound IgE produce histamines, leukotrienes, cytokines and chemokines [2]. However, more recent studies have demonstrated that basophils can be activated by an array of stimuli in both IgE- dependent and -independent manners. Further, basophils are capable of secreting a variety of cytokines including IL-4, IL-6, IL13 , tumor necrosis factor (TNF) $\alpha$ and thymic stromal lymphopoietin (TSLP) [2]. The following section will summarize our current knowledge of the stimuli that activate basophils and the resulting effector molecules they secrete.

\section{IgE-, IgG- and IgD-mediated Activation of Basophils}

As discussed above, basophils produce pre-formed mediators such as histamines and leukotrienes in response to FceRI crosslinking via surface bound IgE. Thus, similar to tissue resident mast cells, basophils are capable of immediately responding to antigens found in the blood [12]. The ability of basophils to rapidly produce pre-formed mediators upon antigen exposure has implicated them as contributors to systemic anaphylaxis in humans [12]. In addition to IgEmediated anaphylaxis, basophils have also been shown to contribute to a novel form of IgE-mediated chronic allergic inflammation in mice [8].

Although basophils are not essential players in the immediate- or late phase- responses that result after multivalent antigens are administered to mice via a subcutaneous injection into the ear, they are required for the chronic inflammation that follows [8]. More specifically, when basophils, 
which represented only $1-2 \%$ of the cellular infiltrate in the ear, were depleted, there was a dramatic reduction in the number of infiltrating eosinophils and neutrophils and a marked reduction in ear thickness [8]. These data suggest that lesion-resident basophils may either directly produce chemokines that recruit inflammatory granulocytes, or produce mediators that indirectly induce the production of chemokines from tissue resident cells. Further, these studies demonstrate the potency of small numbers of basophils and illustrate their ability to significantly influence inflammatory responses.

In addition to IgE-mediated activation, basophils can also be activated by binding IgG or IgD antibodies [7, 13]. For example, basophils produce platelet activating factor (PAF) during an IgG1-mediated alternative pathway of anaphylaxis [7]. Specifically, when C57BL/6 mice and mast cell deficient $\mathrm{Kit}^{\mathrm{W}-\mathrm{sh} / \mathrm{W} \text {-sh }}$ mice were sensitized with Penicillin V (Pen V) and subsequently challenged with Pen V-bovine serum albumin, both wild-type and mast cell-deficient mice developed systemic anaphylaxis. Depleting basophils prior to secondary challenge proved to be protective, implicating basophils as essential mediators of IgG1-mediated anaphylaxis in this model [7]. Further, basophils were also shown to be the dominant cell type that captured Pen $\mathrm{V}$ in an IgG1dependent manner [7].

Human basophils are also capable of binding and being activated by IgD, a class of antibody expressed by mature B cells before class switching has occurred [13]. Although the functions of $\operatorname{IgD}$ remain unclear, it is highly expressed in the upper respiratory tract of humans and can bind the bacteria Haemophilus influenzae and Moraxella catarrhalis [13]. IgD-activated basophils produce a unique array of molecules including IL-4, B cell-activating factor, and a broad spectrum of antimicrobial peptides, but do not release histamine. Further, supernatants from IgD-activated basophils promoted B cell class switching to $\operatorname{IgA}$ and $\operatorname{IgD}$ and were able to prevent the replication of both $H$. influenzae and $M$. catarrhalis [13]. Collectively, these data suggest that IgD and basophils may be critical in providing protection against pathogens found in the human respiratory tract.

\section{Cytokine Activation of Basophils: the Role of IL-3, IL-18 and IL-33}

Although baseline levels of basophils are relatively low, basophil populations significantly expand following exposure to Nippostrongylus brasiliensis and Strongyloides venezuelensis, two models of murine hookworm infection [14]. The expansion of basophil populations in response to both $N$. brasiliensis and $S$. venezuelensis is IL-3-dependent, indicating that IL-3 is an important basophil growth factor [14]. The ability of IL-3 to expand basophil populations was further demonstrated by studies showing that IL-3 promotes the development of basophils in vivo and the differentiation of basophils from bone marrow (BM) precursors in vitro $[15$, 16]. Further, IL-3 can augment the basophil-specific secretion of IL-4 and IL-13 after IgE-mediated activation and is also necessary for the migration of basophils to the draining lymph nodes (LNs) following N. brasiliensis infection [1719]. Collectively, these data have prompted the hypothesis that the development and activation of basophils is highly dependent on IL-3-IL-3R signaling. However, IL-3 is not necessary for the emergence of baseline numbers of basophils in vivo, suggesting that other cytokines or bioactive molecules may be capable of regulating basophil development $[14,15]$. For example, a recent study suggests that basophil development may arise in the periphery from an IL25-dependent multipotent cell population termed MPP ${ }^{\text {type2 }}$ cells, although the role of IL-3 in this response has not been examined [20]. Further, the cytokines IL-18 and IL-33 have been shown to be capable of activating basophils, although their influence on basophil development has not been examined in detail $[15,21,22]$.

IL-18 and IL-33 are members of the IL-1 cytokine family and contribute to Th2 cytokine production and Th2 cytokinemediated inflammation [23-27]. Consistent with their connection to Th2 cytokine-mediated immunity and inflammation, IL-18 and IL-33 are both capable of directly activating or augmenting the activation of basophils. For example, murine BM-derived basophils produce more IL-4 and IL-13 when activated with IL-3 and IL-18 than when they are activated with IL-3 alone [25]. IL-18 is also capable of enhancing IL-4, histamine production and basophil survival in vivo $[21,25]$. Similar to IL-18, IL-33 is also capable of enhancing IL-3-induced secretion of IL-4 and IL-13 from basophils [21]. Further, IL-33 alone can activate human basophils to produce IL-4, IL-5, IL-6 and IL-13 [28]. Collectively, these data demonstrate that cytokines other than IL-3 may be capable of regulating basophil development and activation.

\section{Direct Activation of Basophils by Antigens}

Although it is well documented that basophils can be activated through antibody- and cytokine-mediated pathways, recent data demonstrated that basophils can be directly activated by antigens. In particular, the house dust mite protease Der $\mathrm{p} 1$ and protease antigens secreted by the hookworm parasite Necator americanus induce the production of IL-4, IL-5 and IL-13 from a human basophil cell line [29]. In addition, papain, a cystein protease allergen, induces the production of IL-4 and IL- 6 from murine BM-derived basophils and also induces the migration of basophils to the draining LN when administered subcutaneously [30]. Critically, the activation of basophils by proteases can occur in the absence of antigen-specific $\operatorname{IgE}$, but requires the protease to be functionally active, suggesting that basophils express receptors that are capable of sensing protease activity [30]. Protease activated receptors (PARs) are a family of receptors that recognize protease activity and have been shown to be expressed on other innate and adaptive immune cells, however there is no evidence to date that human or murine basophils express PARs [31]. Collectively these studies suggest that basophils may express PAR-like receptors and/or possess other innate mechanisms by which they recognize protease antigens.

\section{Monitoring Basophil Activation}

As mentioned above, basophils can be activated by a wide range of endogenous and exogenous signals. The following section will summarize the surface marker phenotypes that are known to be associated with basophil activation. 
Activated basophils accumulate in peripheral tissues during late-phase and chronic allergic responses as well as in response to parasitic infections $[3,4,8]$. The ability to identify activated basophils in various tissue microenvironments by flow cytometry has proven to be an invaluable tool. Several surface markers have now been shown to be upregulated by human and murine basophils in response to various stimuli. Analysis of human basophils identified that the expression of CD63, a member of the transmembrane 4 superfamily, is elevated on basophils that are activated in response to allergens including pollens and venom [32, 33]. CD63 is anchored in the basophil granule membrane and upon activation and degranulation gains access to the cell surface [34, 35]. Although CD63 is upregulated on basophils after activation, its level of expression can vary and it was not significantly upregulated on basophils isolated from individuals with drug allergies [36]. In addition, the high expression of CD63 on activated platelets that are capable of adhering to basophils, further complicates its use as an activation marker during flow cytometry studies [37]. Therefore, although useful, CD63 is not considered the most sensitive or accurate marker of basophil activation [36].

Another human basophil marker, the type IItransmembrane protein CD203c has been shown to be upregulated in response to FceRI crosslinking [38]. CD203c shows higher expression levels after basophil activation than CD63 and is upregulated rapidly after allergen challenge. Thus, CD203c is considered a reliable marker that is differentially expressed on resting and activated basophils [38, 39]. Whether co-expression of CD63 and CD203c can be used in combination to provide a more comprehensive panel of activation markers remains to be tested [36].

Perhaps the most promising activation marker on human basophils is the disulfide-linked homodimer CD69. Human basophils exhibit elevated expression of CD69 in response to IL-3 and to a lesser extent in response to antigen stimulation [39-41]. Thus, the induction of CD69 in response to cytokines and antigens suggests it may be a reliable activation marker. Furthermore, CD69 appears to be an activation marker expressed on murine basophils. For example, CD69 expression is elevated on activated basophils in the lung after infection with $N$. brasiliensis [4]. Although CD69 shows promise as a murine basophil activation marker, further studies are necessary to determine its reliability. Few studies have focused on identifying activation markers for murine basophils. In fact, CD200R, an inhibitory receptor that belongs to the immunoglobulin superfamily, is the only recognized activation marker for murine basophils. CD200R is transiently upregulated in response to both IgE-dependent and -independent stimuli [42]. However, the transient nature of CD200R upregulation may make it difficult to use when tracking basophil activation in vivo during allergic inflammation and in response to helminth infections.

\section{Antigen Presenting Cell Functions of Basophils: a New Link Between Innate and Adaptive Immunity}

It has long been appreciated that basophils are potent producers of the Th2 cytokines IL-4 and IL-13 and that they accumulate at sites of inflammation after exposure to allergens or helminth parasites. However, recent evidence now suggests that basophils serve as liaisons between innate and the adaptive immune system. It is now known that IL-4 producing basophils express major histocompatibility complex (MHC) class II, co-stimulatory molecules and migrate to draining LNs where they come in contact with naïve $\mathrm{CD} 4^{+} \mathrm{T}$ cell populations [30, 43-45]. Further, basophils are capable of capturing antigen in an IgE-dependent manner and can directly induce $\mathrm{CD}^{+} \mathrm{T}$ cell proliferation and Th2 cytokine production [45]. The following section will summarize these findings and highlight the role of basophils as a link between innate and adaptive forms of Th2 cytokine-dependent immunity and inflammation.

\section{Basophil-derived IL-4 promotes Th2 Cell Responses}

The ability of DCs to promote Th1 cell differentiation during viral, bacterial or protozoan infections via their activation through pattern recognition receptors (PRRs), up regulation of co-stimulatory molecules and production of IL12 and other proinflammatory cytokines is well-documented [46-48]. However, the inability of DCs to produce IL-4 has provoked questions regarding how DC populations are sufficient to induce and sustain $\mathrm{CD} 4^{+} \mathrm{Th} 2$ cell differentiation [4749]. These questions formed the basis for a hypothesis that an innate cell population capable of producing IL-4 may be required to initiate and/or maintain optimal Th2 cell differentiation [49]. Several innate cell populations can produce IL4 , including NKT cells, eosinophils, mast cells and basophils, and thus are capable of providing a link between innate and adaptive Th2 cytokine responses [49].

There is strong evidence from previous studies that directly implicates basophils as a likely candidate cell population capable of initiating Th2 cell development. For example, basophils isolated from the spleen, liver and BM are capable of promoting IL-4-dependent Th2 cell development when added to cultures containing naïve $\mathrm{T}$ cells, DCs and antigen $[50,51]$. Further, interferon regulatory factor (IRF) $2^{-/-}$mice, which exhibit substantially increased basophil populations in the periphery, were shown to have enhanced steady state levels of $\mathrm{CD}^{+} \mathrm{Th} 2$ cells [50]. In vitro studies demonstrated that basophils were the dominant population of IL-4 producing cells in the spleen of IRF2 ${ }^{-/-}$mice capable of initiating Th2 cytokine production from $\mathrm{CD}^{+} \mathrm{T}$ cells [50]. Collectively, these studies illustrated the potential of basophils to promote Th2 cytokine-dependent immune responses.

\section{Basophil Migration Into Lymph Nodes}

Recent studies demonstrated that IL-4/eGFP ${ }^{+}, \mathrm{MHC}$ class $\mathrm{II}^{+}$basophils migrate to the draining LNs following exposure to papain, Schistosoma mansoni eggs and $N$. brasiliensis infection [19, 30, 44]. Although basophils are only transiently present in the LNs, these data suggest that basophils are capable of directly interacting with LN-resident $\mathrm{CD}^{+} \mathrm{T}$ cells, B cells and DC populations post-challenge. Critically, it was also shown that depleting basophil populations prior to papain challenge prevented the accumulation of IL4/eGFP expressing $\mathrm{CD}^{+} \mathrm{T}$ cells, suggesting an essential role for basophils in the induction of papain-induced Th2 cell differentiation [30].

Although the mechanisms by which basophils enter LNs and their importance in inducing $\mathrm{CD}^{+} \mathrm{T}$ cell differentiation remain unknown, a recent study suggests that IL-3 may be a critical regulator of basophil LN recruitment. Infection with 
$N$. brasiliensis induced the accumulation of basophils to the mediastinal LNs on days 4 and 10 post-infection [19]. Interestingly, basophils failed to accumulate in the LNs in the absence of IL-3-IL-3R signaling. In addition, the loss of basophils in the LNs of IL- $3^{-/-}$mice did not prevent the induction of Th2 cell differentiation, suggesting that in the specific case of $N$. brasiliensis, basophils are not essential for the induction of Th2 cytokine-mediated immunity [19]. The basophil-independent nature of Th2 cell differentiation in this model is perhaps not surprising considering that multiple redundant mechanisms for Th2 cell development are initiated post- $N$. brasiliensis infection. For example, it has been shown that Th2 cell development post- $N$. brasiliensis infection can also occur in the absence of STAT6 or the combined absence of IL-5, IL-9 and IL-13 signaling [52, 53]. These findings also highlight that the potential contribution of basophils to local IL-4 production or antigen presentation may depend on the nature of the stimulus, the strength of signal and the route of antigen or allergen exposure.

\section{APC Functions of Basophils}

In a papain allergen model, an OVA/Alum allergic inflammation model and following Trichuris infection, it has been demonstrated that restricting MHC class II expression to DC populations is insufficient to induce optimal Th2 cell differentiation in vivo [43-54]. Further, depleting basophil populations prior to papain challenge and Trichuris infection significantly reduced the magnitude of Th2 cytokine responses, suggesting that basophils could be critical participants in the induction of optimal Th2 cytokine responses in vivo $[43,44]$. These observations were further strengthened by gain of function studies illustrating that the transfer of antigen-loaded MHC class $\mathrm{II}^{+}$basophils into $\mathrm{CIITA}^{-/-}$mice, which have deficiencies in MHC class II expression, was sufficient to induce papain-specific Th2 cell differentiation [43]. In addition, the adoptive transfer of IL-4/eGFP ${ }^{+}$MHC class $\mathrm{II}^{+}$basophils was capable of augmenting S. mansoni egg-induced Th2 cell differentiation in vivo [44]. The function of basophils as APCs was further demonstrated by in vitro studies demonstrating that basophils are capable of taking up antigens in an FceRI-dependent manner and could present them via MHC class II [45]. Further, three independent studies demonstrated that basophils can induce OVAspecific $\mathrm{CD} 4^{+} \mathrm{T}$ cell proliferation and $\mathrm{Th} 2$ cytokine production in the absence of other APC populations in vitro [4345].

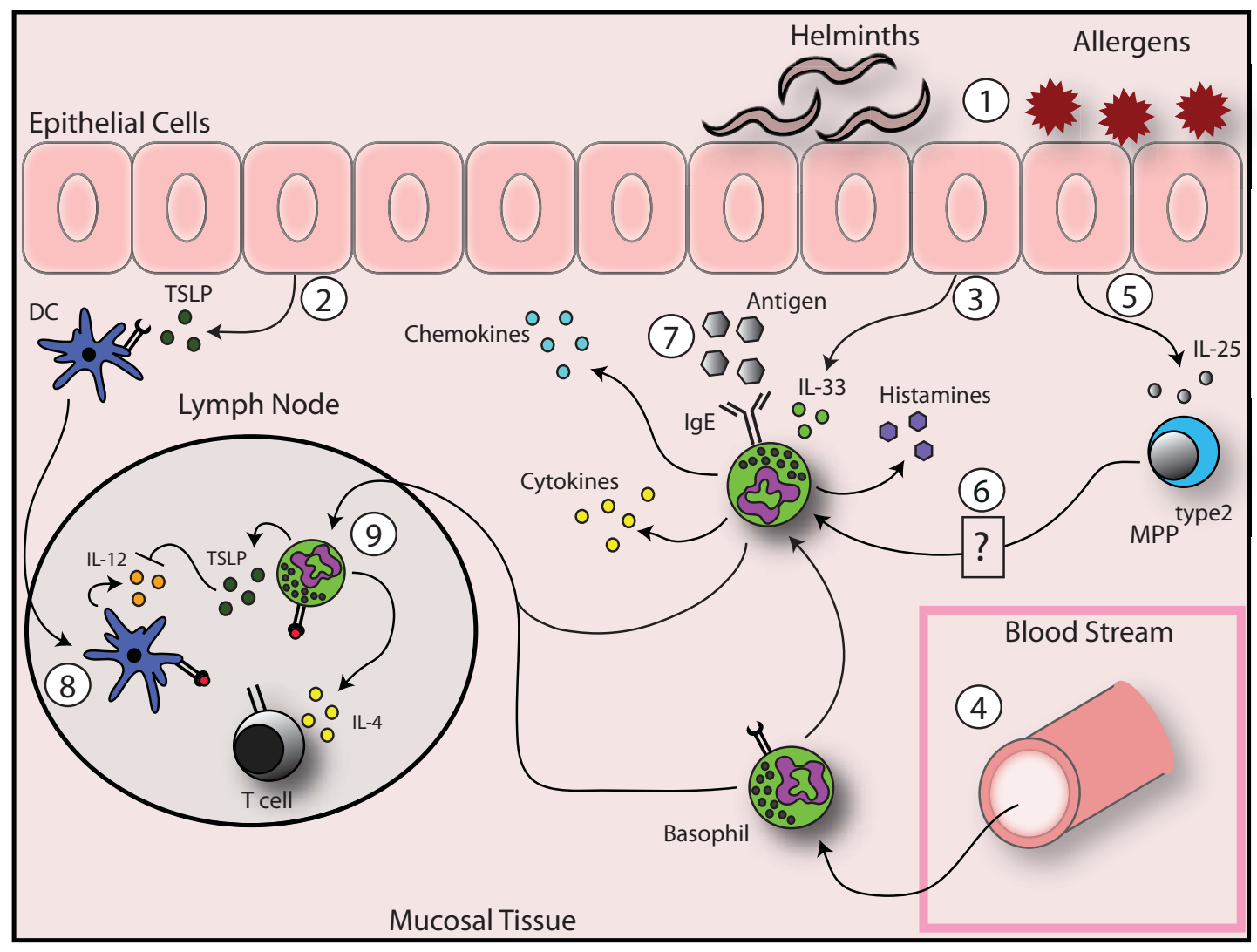

Fig. (1). Basophil activation, migration and effector function. Following exposure to helminth parasites or allergens at barrier surfaces 1 , the epithelial derived cytokines TSLP, IL-33 and IL-25 are produced. TSLP can act directly on tissue-resident DC populations to prevent their upregulation of costimulatory molecules and IL-12/23p40 production, creating an environment permissive for Th2 cell differentiation 2. IL-33 can directly activate basophil populations 3 that have been recruited to the site of challenge from the blood stream $\mathbf{4}$, while IL-25 has been shown to elicit a multipotent progenitor cell population termed $\mathrm{MPP}^{\text {type2 }} \mathbf{5}$. MPP ${ }^{\text {type2 }}$ cells have the capacity to differentiate into basophils and may contribute to the expansion of basophil populations at the site of challenge $\boldsymbol{6}$. Basophils can also be activated by antigen via surface bound $\operatorname{IgE} \boldsymbol{\nabla}$ and are capable of producing effector molecules including histamines, cytokines and chemokines. MHC class II expressing DCs 8 and basophils $\boldsymbol{\Theta}$ can migrate to the draining lymph node where they come in contact with naive CD4 $4^{+} \mathrm{T}$ cell populations. Once in the LN, basophil-derived TSLP and IL-4 can limit the production of IL-12/23p40 by DC populations and can promote the differentiation of $\mathrm{CD}^{+} \mathrm{T}$ cells. Antigen presentation by both DCs and basophils may act cooperatively to expand Th2 cell populations. 
Collectively, these studies demonstrated that basophils express MHC class II and co-stimulatory molecules, endocytose antigen and can promote $\mathrm{CD} 4^{+} \mathrm{T}$ cell proliferation and Th2 cell differentiation both in vitro and in vivo. These data also support the hypothesis that basophils provide a link between innate and adaptive forms of Th2 cytokine responses and significantly advance our understanding of how Th2 cytokine-dependent immunity and inflammation are initiated. These data suggest that some basophil populations may share functional characteristics with DCs, expressing MHC class II and costimulatory molecules, endocytosing soluble antigens, migrating to draining LNs and producing cytokines that can promote the initiation and propagation of Th2 cells (Fig. 1. 4, 0,0).

The ability of basophils to function as APCs in the absence of DCs does not preclude the possibility that DCs and basophils may function in concert to initiate Th2 cell development and propagation. There are several potential pathways through which basophils and DCs may act cooperatively to induce Th2 cytokines responses. Basophils may act as IL-4 producing accessory cells that promote the differentiation of T cells previously activated by DCs. Alternatively, basophils and DCs may present antigens to T cells in parallel (Fig. 18,9). In this scenario, basophils could augment antigen presentation, increase $\mathrm{T}$ cell proliferation and provide the IL-4 necessary for optimal Th2 cell differentiation. In the context of this model, basophils may further enhance Th2 cell development by producing TSLP that inhibits the ability of DCs to produce IL-12/23p40 and upregulate costimulatory molecules (Fig. 19). Further studies will be required to directly assess the relative contributions of basophils and DCs in promoting Th2 cytokine responses in models of allergy and helminth infection. Understanding the mechanisms through which basophils cooperatively interact with DCs and other professional antigen presenting cells may provide new targets to limit or promote the development of Th2 celldependent immune responses.

\section{SUMMARY AND FUTURE DIRECTIONS}

Although relatively ignored for almost a century, recent studies have fundamentally altered our understanding of the functional biology of basophils. It is now appreciated that basophils can be activated by an array of signals including those mediated by cytokines, antibodies and directly by antigens themselves. Once activated, MHC class $\mathrm{II}^{+}$basophils can migrate into LNs, present antigen to naïve $\mathrm{CD} 4^{+} \mathrm{T}$ cells and induce Th2 cell differentiation. The ability of basophils to function as APCs and their emerging central role in initiating and regulating Th2 cytokine responses suggest that further exploring the factors that regulate basophil development and function may lead to therapeutic strategies for the treatment of Th2-cytokine induced inflammation.

\section{ACKNOWLEDGEMENTS}

We thank members of the Artis laboratory for constructive discussions and critical reading of this article. Work in the Artis lab is supported by the National Institutes of Health (AI61570, AI074878 and AI083480 to D.A., KirschsteinNRSA postdoctoral fellowship AI085828 to M.S.). Burroughs Wellcome Fund (D.A.), a National Institute of Diabetes and Digestive Kidney Disease Center Grant (P30
DK50306) and pilot grants from the University of Pennsylvania (URF, VCID and PGI) (to D.A.).

\section{REFERENCES}

[1] Ishizaka T, De Bernardo R, Tomioka H, Lichtenstein LM, Ishizaka $\mathrm{K}$. Identification of basophil granulocytes as a site of allergic histamine release. J Immunol 1972;108(4):1000-8.

[2] Schroeder JT. Basophils beyond effector cells of allergic inflammation. Adv Immunol 2009;101:123-61.

[3] Min B, Prout M, Hu-Li J, Zhu J, Jankovic D, Morgan ES, et al. Basophils produce IL-4 and accumulate in tissues after infection with a Th2-inducing parasite. J Exp Med 2004; 200(4): 507-17.

[4] Voehringer D, Shinkai K, Locksley RM. Type 2 immunity reflects orchestrated recruitment of cells committed to IL-4 production. Immunity 2004; 20(3): 267-77.

[5] Gessner A, Mohrs K, Mohrs M. Mast cells, basophils, and eosinophils acquire constitutive IL-4 and IL-13 transcripts during lineage differentiation that are sufficient for rapid cytokine production. J Immunol 2005; 174(2): 1063-72.

[6] Denzel A, Maus UA, Rodriguez Gomez M, et al. Basophils enhance immunological memory responses. Nat Immunol 2008; 9(7): $733-42$.

[7] Tsujimura $\mathrm{Y}$, Obata K, Mukai K, et al. Basophils play a pivotal role in immunoglobulin-G-mediated but not immunoglobulin-Emediated systemic anaphylaxis. Immunity 2008; 28(4): 581-9.

[8] Obata K, Mukai K, Tsujimura Y, et al. Basophils are essential initiators of a novel type of chronic allergic inflammation. Blood 2007; 110(3): 913-20.

[9] Kojima T, Obata K, Mukai K, et al. Mast cells and basophils are selectively activated in vitro and in vivo through CD200R3 in an IgE-independent manner. J Immunol 2007; 179(10): 7093-100.

[10] Kawakami T, Galli SJ. Regulation of mast-cell and basophil function and survival by IgE. Nat Rev Immunol 2002; 2(10): 773-86.

[11] Tschopp CM, Spiegl N, Didichenko S, et al. Granzyme B, a novel mediator of allergic inflammation: its induction and release in blood basophils and human asthma. Blood 2006; 108(7): 2290-9.

[12] Golden DB. What is anaphylaxis? Curr Opin Allergy Clin Immunol 2007; 7(4): 331-6.

[13] Chen K, Xu W, Wilson M, et al. Immunoglobulin D enhances immune surveillance by activating antimicrobial, proinflammatory and B cell-stimulating programs in basophils. Nat Immunol 2009; 10(8): 889-98.

[14] Lantz CS, Min B, Tsai M, Chatterjea D, Dranoff G, Galli SJ. IL-3 is required for increases in blood basophils in nematode infection in mice and can enhance IgE-dependent IL-4 production by basophils in vitro. Lab Invest 2008; 88(11): 1134-42.

[15] Lantz CS, Boesiger J, Song CH, et al. Role for interleukin-3 in mast-cell and basophil development and in immunity to parasites. Nature 1998; 392(6671): 90-3.

[16] Ohmori K, Luo Y, Jia Y, et al. IL-3 induces basophil expansion in vivo by directing granulocyte-monocyte progenitors to differentiate into basophil lineage-restricted progenitors in the bone marrow and by increasing the number of basophil/mast cell progenitors in the spleen. J Immunol 2009; 182(5): 2835-41.

[17] Gibbs BF, Haas H, Falcone FH, et al. Purified human peripheral blood basophils release interleukin-13 and preformed interleukin-4 following immunological activation. Eur J Immunol 1996; 26(10): 2493-8.

[18] MacGlashan DJr., White JM, Huang SK, Ono SJ, Schroeder JT, Lichtenstein LM. Secretion of IL-4 from human basophils. The relationship between IL-4 mRNA and protein in resting and stimulated basophils. J Immunol 1994; 152(6): 3006-16.

[19] Kim S, Prout M, Ramshaw H, Lopez AF, LeGros G, Min B. Cutting edge: Basophils are transiently recruited into the draining lymph nodes during helminth infection via IL-3, but infectioninduced Th2 immunity can develop without basophil lymph node recruitment or IL-3. J Immunol 184(3): 1143-7.

[20] Saenz SA, Siracusa MC, Perrigoue JG, et al. IL25 elicits a multipotent progenitor cell population that promotes $\mathrm{T}(\mathrm{H}) 2$ cytokine responses. Nature 2010; 464(7293): 1362-6.

[21] Kroeger KM, Sullivan BM, Locksley RM. IL-18 and IL-33 elicit Th2 cytokines from basophils via a MyD88- and p38alphadependent pathway. J Leukoc Biol 2009; 86(4): 769-78. 
[22] Pecaric-Petkovic T, Didichenko SA, Kaempfer S, Spiegl N, Dahinden CA. Human basophils and eosinophils are the direct target leukocytes of the novel IL-1 family member IL-33. Blood 2009; 113(7): 1526-34.

[23] Saito H, Matsumoto K, Okumura S, et al. Gene expression profiling of human mast cell subtypes: an in silico study. Allergol Int 2006; 55(2): 173-9.

[24] Sasaki Y, Yoshimoto T, Maruyama H, et al. IL-18 with IL-2 protects against Strongyloides venezuelensis infection by activating mucosal mast cell-dependent type 2 innate immunity. J Exp Med 2005; 202(5): 607-16.

[25] Yoshimoto T, Tsutsui H, Tominaga K, et al. IL-18, although antiallergic when administered with IL-12, stimulates IL-4 and histamine release by basophils. Proc Natl Acad Sci U S A 1999; 96(24): 13962-6.

[26] Helmby H, Grencis RK. IL-18 regulates intestinal mastocytosis and Th2 cytokine production independently of IFN-gamma during Trichinella spiralis infection. J Immunol 2002; 169(5): 2553-60.

[27] Schmitz J, Owyang A, Oldham E, et al. IL-33, an interleukin-1-like cytokine that signals via the IL-1 receptor-related protein ST2 and induces $\mathrm{T}$ helper type 2-associated cytokines. Immunity 2005; 23(5): 479-90

[28] Smithgall MD, Comeau MR, Yoon BR, Kaufman D, Armitage R, Smith DE. IL-33 amplifies both Th1- and Th2-type responses through its activity on human basophils, allergen-reactive Th2 cells, iNKT and NK cells. Int Immunol 2008; 20(8): 1019-30.

[29] Phillips C, Coward WR, Pritchard DI, Hewitt CR. Basophils express a type 2 cytokine profile on exposure to proteases from helminths and house dust mites. J Leukoc Biol 2003; 73(1): 16571.

[30] Sokol CL, Barton GM, Farr AG, Medzhitov R. A mechanism for the initiation of allergen-induced $\mathrm{T}$ helper type 2 responses. Nat Immunol 2008; 9(3): 310-8.

[31] Falcone FH, Morroll S, Gibbs BF. Lack of protease activated receptor (PAR) expression in purified human basophils. Inflamm Res 2005; 54 Suppl 1: S13-4.

[32] Paris-Kohler A, Demoly P, Persi L, Lebel B, Bousquet J, Arnoux B. In vitro diagnosis of cypress pollen allergy by using cytofluorimetric analysis of basophils (Basotest). J Allergy Clin Immunol 2000; 105(2 Pt 1): 339-45.

[33] Sainte-Laudy J, Boumediene A, Touraine F, et al. Use of both CD63 up regulation and IgE down regulation for the flow cytometric analysis of allergen induced basophil activation. Definition of an activation index. Inflamm Res 2007; 56(7): 291-6.

[34] Knol EF, Mul FP, Jansen H, Calafat J, Roos D. Monitoring human basophil activation via CD63 monoclonal antibody 435. J Allergy Clin Immunol 1991; 88(3 Pt 1): 328-38.

[35] Fureder W, Agis H, Sperr WR, Lechner K, Valent P. The surface membrane antigen phenotype of human blood basophils. Allergy 1994; 49(10): 861-5.

[36] Boumiza R, Debard AL, Monneret G. The basophil activation test by flow cytometry: recent developments in clinical studies, standardization and emerging perspectives. Clin Mol Allergy 2005; 3: 9 .
[37] Goschnick MW, Jackson DE. Tetraspanins-structural and signalling scaffolds that regulate platelet function. Mini Rev Med Chem 2007; 7(12): 1248-54.

[38] Buhring HJ, Seiffert M, Giesert C, et al. The basophil activation marker defined by antibody 97A6 is identical to the ectonucleotide pyrophosphatase/phosphodiesterase 3. Blood 2001; 97(10): 3303-5.

[39] Gober LM, Eckman JA, Sterba PM, et al. Expression of activation markers on basophils in a controlled model of anaphylaxis. J Allergy Clin Immunol 2007; 119(5): 1181-8.

[40] Yoshimura C, Yamaguchi M, Iikura M, et al. Activation markers of human basophils: CD69 expression is strongly and preferentially induced by IL-3. J Allergy Clin Immunol 2002; 109(5): 817-23.

[41] Suzukawa M, Komiya A, Yoshimura-Uchiyama C, et al. IgE- and FcepsilonRI-mediated enhancement of surface CD69 expression in basophils: role of low-level stimulation. Int Arch Allergy Immunol 2007; 143 Suppl 1: 56-9.

[42] Torrero MN, Larson D, Hubner MP, Mitre E. CD200R surface expression as a marker of murine basophil activation. Clin Exp Allergy 2009; 39(3): 361-9.

[43] Sokol CL, Chu NQ, Yu S, Nish SA, Laufer TM, Medzhitov R. Basophils function as antigen-presenting cells for an allergeninduced T helper type 2 response. Nat Immunol 2009; 10(7): 71320 .

[44] Perrigoue JG, Saenz SA, Siracusa MC, et al. MHC class IIdependent basophil-CD4+ T cell interactions promote $\mathrm{T}(\mathrm{H}) 2$ cytokine-dependent immunity. Nat Immunol 2009; 10(7): 697-705.

[45] Yoshimoto T, Yasuda K, Tanaka H, et al. Basophils contribute to $\mathrm{T}(\mathrm{H}) 2-\mathrm{IgE}$ responses in vivo via IL-4 production and presentation of peptide-MHC class II complexes to CD4+ T cells. Nat Immunol 2009; 10(7): 706-12.

[46] Schnare M, Barton GM, Holt AC, Takeda K, Akira S, Medzhitov R. Toll-like receptors control activation of adaptive immune responses. Nat Immunol 2001; 2(10): 947-50.

[47] Fearon DT, Locksley RM. The instructive role of innate immunity in the acquired immune response. Science 1996; 272(5258): 50-3.

[48] Yamamoto M, Sato S, Hemmi H, et al. Role of adaptor TRIF in the MyD88-independent toll-like receptor signaling pathway. Science 2003; 301(5633): 640-3.

[49] Min B, Le Gros G, Paul WE. Basophils: a potential liaison between innate and adaptive immunity. Allergol Int 2006; 55(2): 99-104

[50] Hida S, Tadachi M, Saito T, Taki S. Negative control of basophil expansion by IRF- 2 critical for the regulation of Th1/Th2 balance. Blood 2005; 106(6): 2011-7.

[51] Oh K, Shen T, Le Gros G, Min B. Induction of Th2 type immunity in a mouse system reveals a novel immunoregulatory role of basophils. Blood 2007; 109(7): 2921-7.

[52] van Panhuys N, Tang SC, Prout M, et al. In vivo studies fail to reveal a role for IL-4 or STAT6 signaling in Th2 lymphocyte differentiation. Proc Natl Acad Sci U S A 2008; 105(34): 12423-8.

[53] Fallon PG, Jolin HE, Smith P, et al. IL-4 induces characteristic Th2 responses even in the combined absence of IL-5, IL-9, and IL-13. Immunity 2002; 17(1): 7-17.

[54] Niu N, Laufer T, Homer RJ, Cohn L. Cutting edge: Limiting MHC class II expression to dendritic cells alters the ability to develop Th2- dependent allergic airway inflammation. J Immunol 2009; 183(3): 1523-7.

(c) Siracusa and Artis; Licensee Bentham Open.

This is an open access article licensed under the terms of the Creative Commons Attribution Non-Commercial License (http://creativecommons.org/licenses/by-nc/3.0/) which permits unrestricted, non-commercial use, distribution and reproduction in any medium, provided the work is properly cited. 